\title{
THE MONOZYGOTIC TWIN HALF-SIB METHOD FOR ANALYSING MATERNAL EFFECTS AND SEX-LINKAGE IN HUMANS
}

\author{
C. S. HALEY, J. L. JINKS and KRYSTYNA LAST \\ Department of Genetics, University of Birmingham, Birmingham B15 2TT \\ Received 25.ix.80
}

\section{SUMMARY}

\begin{abstract}
The influence of maternal effects and sex-linked loci upon the statistics derived from an analysis of the families of monozygotic twins (the so called MZ half-sib design of Nance and Corey, 1976) is examined. If, as in the published analyses, the progeny sexes are considered jointly, maternal effects and sex-linkage may be confused. In the absence of sex-linkage a comparison of maternal and paternal half-sib covariances will often be a more powerful test for maternal effects than the conventional comparison of maternal and paternal parent-offspring covariances. If the progeny sexes are considered separately the analysis of sex-linkage presents no fundamental problems.
\end{abstract}

\section{INTRODUCTION}

IN experimental organisms the effects of maternal inheritance may be unambiguously distinguished from those of sex-linkage by the use of reciprocal crosses. In hermaphroditic species the same individual may be used as both male and female parent to produce reciprocal crosses between pairs of individuals. In other species male and female sibs from genetically homogeneous families can be used to produce reciprocal crosses between pairs of families. In man neither strategy for making reciprocal crosses is possible since the only genetically homogeneous families are pairs of identical twins. Detecting maternal effects and sex-linkage in man and discriminating between them is therefore difficult and indirect.

Attempts to produce diagnostic tests in man have concentrated on standard relationships such as parent-offspring, full-sib, half-sib and cousin correlations (Mather and Jinks, 1963; Rao, Morton and Yee, 1974; Robson, 1955; Bock and Kolakowski, 1973). Recently, however, Nance and Corey (1976) added to these a new approach "the Monozygotic Twin (MZ) half-sib method" which they suggest provides unambiguous tests for maternal effects and sex-linkage.

In this paper we shall explore the effects of maternal inheritance and sex-linkage on the statistics yielded by Nance and Corey's approach and examine the consequences of making assumptions about the nature of these effects so that we can identify potential difficulties in interpretation that could arise in practice. At the same time we shall consider the relative sensitivities and reliabilities of the $\mathrm{MZ}$ half-sib method and previous methods for detecting and correctly identifying maternal inheritance and sex-linkage.

\section{THE BASIC MODEL}

The MZ half-sib design of Nance and Corey (1976) requires the ascertainment of the offspring and spouses of monozygotic twins. The structure 

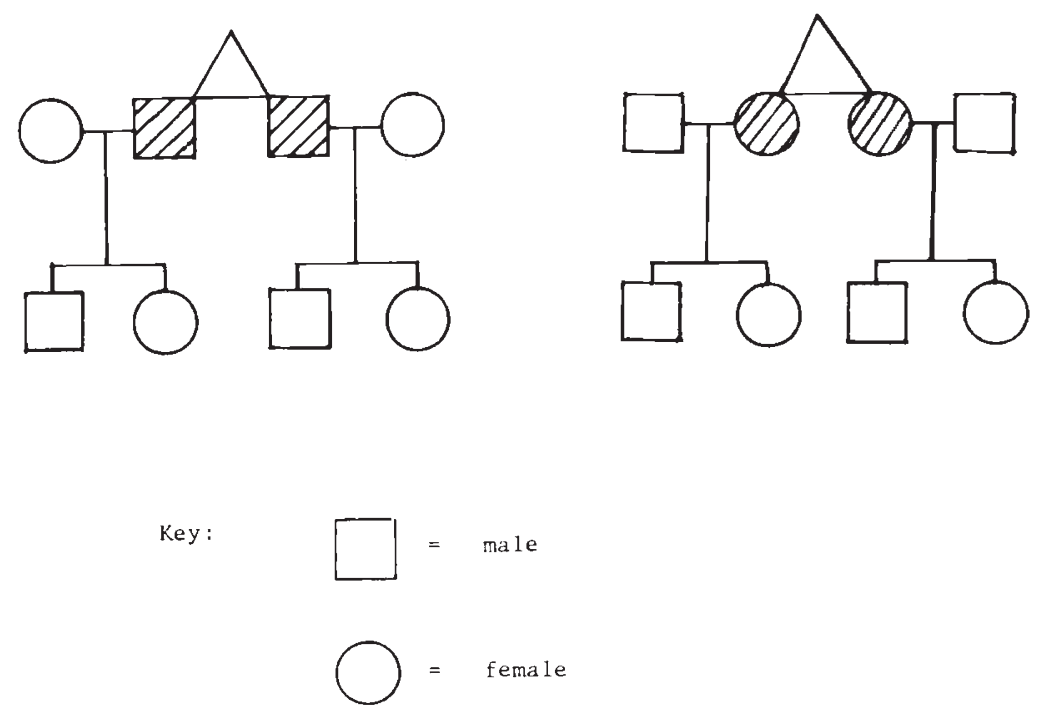

FIG. 1.-The idealized family structure of the data showing one of each of paternal and maternal half-sibships respectively. The twins are shown shaded.

of the data is shown in fig. 1. A hierarchical analysis of variance of the offspring from this design yields three mean squares:

$\mathrm{MS}_{W}$ : between progeny within full-sib families

$\mathrm{MS}_{B}$ : between the two families of full-sibs produced by pairs of monozygotic twins (these are genetically half-sib families).

$\mathrm{MS}_{A}$ : among genetically half-sib families.

Three corresponding components of variance $\left(\sigma_{w}^{2}, \sigma_{b}^{2}\right.$ and $\left.\sigma_{a}^{2}\right)$ can be derived from these mean squares. These components are genetically equivalent to the within family variance, the full-sib minus the half-sib covariance and the half-sib covariance, respectively, of the standard experimental mating designs. The expectations, as given by Mather and Jinks (1971), for these components in the presence of additive and dominance genetical variation and environmental variation are:

$$
\begin{aligned}
\sigma_{w}^{2} & =\frac{1}{4} D_{R}+\frac{3}{16} H_{R}+E_{W} \\
\sigma_{b}^{2} & =\frac{1}{8} D_{R}+\frac{1}{16} H_{R}+E_{B}^{\prime} \\
\sigma_{a}^{2} & =\frac{1}{8} D_{R}+E_{B}^{\prime \prime}
\end{aligned}
$$

where $D_{R}$ is additive genetical component, $H_{R}$ is the dominance genetical component, $E_{W}$ is the within family environmental component, $E_{B}^{\prime}$ is the common environmental component of full-sibs, $E_{B}^{\prime \prime}$ is the common environmental component of genetically half-sib families.

In addition to data from the offspring of the $\mathrm{MZ}$ twins, further data are available from the analysis of the twins themselves and their covariance with their progeny. The expectations of the mean squares and covariances are 
only valid if environmental influences have contributed equally to the variation in both parents and progeny. This being the case, the expectations of the components of variance derived from an analysis of variance within and between $\mathrm{MZ}$ twin parents ( $\sigma_{w}^{2}$ and $\sigma_{b}^{2}$ respectively) and the expectation of the parent-offspring covariance $\left(W_{P O}\right)$ are:

$$
\begin{aligned}
\sigma_{b}^{2} & =\frac{1}{2} D_{R}+\frac{1}{4} H_{R}+E_{B}^{\prime}+E_{B}^{\prime \prime} \\
\sigma_{w}^{2} & =E_{W} \\
W_{P O} & =\frac{1}{4} D_{R} .
\end{aligned}
$$

Note that this model, unlike that of Nance and Corey (1976), assumes that $E_{B}^{\prime}$ and $E_{B}^{\prime \prime}$ only contribute to the similarity of progeny within families and within half-sibships and not to the similarity with their parents who were raised in different sibships.

\section{Maternal EFFECTS}

Maternal effects cannot arise unless a mother makes a contribution to her progeny's phenotype over and above that which results from the genes she contributes to the progeny's genotype. This additional contribution can have many immediate causes but if any of them has a component attributable to the action of maternal chromosomal genes they can be analysed by the methods of biometrical genetics (Mather and Jinks, 1971). We shall consider two models. Firstly, where the genes responsible for the maternal effect influence the same character in the progeny and the parent i.e., the same loci are mediating both a direct effect through the progeny's genotype and an indirect maternal effect. This is the model explored by Kempthorne (1957) and we shall refer to it as the one character model. Secondly, where the loci governing the maternal effect are influencing some characteristic of the mother other than that which is affected in the progeny, i.e., different and independent sets of loci mediate the direct effect of the progeny's genotype and the maternal effect on the character displaying maternal inheritance. At its simplest this model involves two characters; we shall, therefore, refer to it as the two character model. The one character and two character models are special cases of a general model in which maternal effects are contributed to both by the same loci and different loci from those which directly influence the character under study through the progeny's own genotype.

If we assume Hardy-Weinberg equilibrium frequencies, the one character model for a single locus with two alleles $A$ and $a$ and frequencies $u$ and $v$ can be written as:

$\begin{array}{lccc}\text { Genotype } & A A & A a & a a \\ \text { Frequency } & u^{2} & 2 u v & v^{2} \\ \text { Direct effect of genotype on progeny } & d & h & -d \\ \text { Effect of mother of that genotype on progeny } & m d & m h & -m d\end{array}$

Summing over all such loci, we can cast the genetical expectations of the components of variance derived from the $\mathrm{MZ}$ half-sib design in terms of six 
parameters: $D_{R}, H_{R}$ (Mather and Jinks, 1971) and

$$
\begin{aligned}
M D_{R} & =\sum_{i} 4 u_{i} v_{i}\left(m d_{i}+\left(v_{i}-u_{i}\right) m h_{i}\right)^{2} \\
M H_{R} & =\sum_{i} 16 u_{i}^{2} v_{i}^{2} m h_{i}^{2} \\
D M D_{R} & =\sum_{i} 4 u_{i} v_{i}\left(d_{i}+\left(v_{i}-u_{i}\right) h_{i}\right)\left(m d_{i}+\left(v_{i}-u_{i}\right) m h_{i}\right) \\
H M H_{R} & =\sum_{i} 16 u_{i}^{2} v_{i}^{2} h_{i} m h_{i} .
\end{aligned}
$$

The contribution of these six parameters to the statistics derived from an analysis of maternal and paternal $\mathrm{MZ}$ half-sibships is shown in table 1 . The total variance, the full-sib covariance and the maternal and paternal parentoffspring covariances are equivalent to those obtained by Kempthorne (1957). It is perhaps worth noting that, on this model, which is the one used

\begin{tabular}{|c|c|c|}
\hline$\sigma_{a m}^{2}$ & Maternal half-sib covariance & $\frac{1}{8} D_{R}+\frac{1}{2} M D_{R}+\frac{1}{4} M H_{R}+\frac{1}{2} D M D_{R}$ \\
\hline$\sigma_{b m}^{2}$ & $\begin{array}{l}\text { Full-sib covariance minus maternal } \\
\text { half-sib covariance }\end{array}$ & $\frac{1}{8} D_{R}+\frac{1}{16} H_{R}$ \\
\hline$\sigma_{w m}^{2}$ & Within family variance & $\frac{1}{4} D_{R}+\frac{3}{16} H_{R}$ \\
\hline \multicolumn{3}{|c|}{ Paternal $M Z$ half sib-ship } \\
\hline$\sigma_{a p}^{2}$ & Paternal half-sib covariance & $\frac{1}{8} D_{R}$ \\
\hline$\sigma_{b p}^{2}$ & $\begin{array}{l}\text { Full-sib covariance minus paternal } \\
\text { half-sib covariance }\end{array}$ & $\begin{array}{l}\frac{1}{8} D_{R}+\frac{1}{16} H_{R}+\frac{1}{2} M D_{R} \\
\quad+\frac{1}{4} M H_{R}+\frac{1}{2} D M D_{R}\end{array}$ \\
\hline$\sigma_{w p}^{2}$ & Within family variance & $\frac{1}{4} D_{R}+\frac{3}{16} H_{R}$ \\
\hline \multicolumn{3}{|l|}{ Full sib-ship } \\
\hline$\sigma_{a}^{2}+\sigma_{b}^{2}$ & Full-sib covariance & $\begin{array}{l}\frac{1}{4} D_{R}+\frac{1}{16} H_{R}+\frac{1}{2} M D_{R}+\frac{1}{4} M H_{R} \\
\quad+\frac{1}{2} D M D_{R}\end{array}$ \\
\hline$\sigma_{a}^{2}+\sigma_{b}^{2}+\sigma_{w}^{2}$ & Total variance & $\begin{array}{l}\frac{1}{2} D_{R}+\frac{1}{4} H_{R}+\frac{1}{2} M D_{R}+\frac{1}{4} M H_{R} \\
\quad+\frac{1}{2} D M D_{R}\end{array}$ \\
\hline$W_{P O M}$ & Maternal parent-offspring covariance & $\frac{1}{4} D_{R}+\frac{1}{4} M D_{R}+\frac{5}{8} D M D_{R}+\frac{1}{8} H M H_{R}$ \\
\hline$W_{P O P}$ & Paternal parent-offspring covariance & $\frac{1}{4} D_{R}+\frac{1}{8} D M D_{R}$ \\
\hline & $D_{R}=\sum_{i} 4 u_{i} v_{i}\left(d_{i}+\left(v_{i}-u_{i}\right) h_{i}\right)^{2}$ & $H_{R}=\sum_{i} 16 u_{i}^{2} v_{i}^{2} h_{i}^{2}$ \\
\hline & $M D_{R}=\sum_{i} 4 u_{i} v_{i}\left(m d_{i}+\left(v_{i}-u_{i}\right) m h_{i}\right)^{2}$ & $M H_{R}=\sum_{i} 16 u_{i}^{2} v_{i}^{2} m h_{i}^{2}$ \\
\hline & \multicolumn{2}{|c|}{$D M D_{R}=\sum_{i} 4 u_{i} v_{i}\left(d_{i}+\left(v_{i}-u_{i}\right) h_{i}\right)\left(m d_{i}+\left(v_{i}-u_{i}\right) m h_{i}\right)$} \\
\hline
\end{tabular}

\section{TABLE 1}

Maternal effects-one character model

Maternal $M Z$ half sib-ship 
by Nance and Corey (1976), the genetical contribution to the paternal parent-offspring covariance is not, as they assume, expected to be twice that to the paternal half-sib covariance.

If we again assume Hardy-Weinberg equilibrium frequencies, the two character model for a single locus, with two alleles, $B$ and $b$, with frequencies $p$ and $q$, that makes no direct contribution to the character being studied in the offspring through its own genotype can be written as:

$\begin{array}{lccc}\text { Genotype } & B B & B b & b b \\ \text { Frequency } & p^{2} & 2 p q & q^{2} \\ \text { Effect of mother of that genotype on progeny } & m d & m h & -m d\end{array}$

Summing over all such loci we can write the genetical expectations of the components of variance derived from the $\mathrm{MZ}$ half-sib design in terms of four parameters: $D_{R}, H_{R}$ and

$$
\begin{aligned}
M D_{R}^{\prime} & =\sum_{i} 4 p_{i} q_{i}\left(m d_{i}+\left(q_{i}-p_{i}\right) m h_{i}\right)^{2} \\
M H_{R}^{\prime} & =\sum_{i} 16 p_{i}^{2} q_{i}^{2} m h_{i}^{2} .
\end{aligned}
$$

The contribution of these four parameters to the statistics derived from an analysis of maternal MZ half-sibships is shown in table 2.

The most instructive way of examining the consequences of the two models for all situations likely to be encountered in practice is to substitute into the statistics in tables 1 and 2 a wide range of possible numerical relationships between $d, h, m d$ and $m h$ and the full range of possible gene

TABLE 2

Maternal effects-two character model

\begin{tabular}{|c|c|c|}
\hline$\sigma_{a m}^{2}$ & Maternal half-sib covariance & $\frac{1}{8} D_{R}+\frac{1}{2} M D_{R}^{\prime}+\frac{1}{4} M H_{R}^{\prime}$ \\
\hline$\sigma_{b m}^{2}$ & $\begin{array}{l}\text { Full-sib covariance minus maternal } \\
\text { half-sib covariance }\end{array}$ & $\frac{1}{8} D_{R}+\frac{1}{16} H_{R}$ \\
\hline$\sigma_{w m}^{2}$ & Within family variance & $\frac{1}{4} D_{R}+\frac{3}{16} H_{R}$ \\
\hline \multicolumn{3}{|c|}{ Paternal $M Z$ half sib-ship } \\
\hline$\sigma_{a p}^{2}$ & Paternal half-sib covariance & $\frac{1}{8} D_{R}$ \\
\hline$\sigma_{b p}^{2}$ & $\begin{array}{l}\text { Full-sib covariance minus paternal } \\
\text { half-sib covariance }\end{array}$ & $\frac{1}{8} D_{R}+\frac{1}{16} H_{R}+\frac{1}{2} M D_{R}^{\prime}+\frac{1}{4} M H_{R}^{\prime}$ \\
\hline$\sigma_{w p}^{2}$ & Within family variance & $\frac{1}{4} D_{R}+\frac{3}{16} H_{R}$ \\
\hline \multicolumn{3}{|l|}{ Full sib-ship } \\
\hline$\sigma_{a}^{2}+\sigma_{b}^{2}$ & Full-sib covariance & $\frac{1}{4} D_{R}+\frac{1}{16} H_{R}+\frac{1}{2} M D_{R}^{\prime}+\frac{1}{4} M H_{R}^{\prime}$ \\
\hline$\sigma_{a}^{2}+\sigma_{b}^{2}+\sigma_{w}^{2}$ & Total variance & $\frac{1}{2} D_{R}+\frac{1}{4} H_{R}+\frac{1}{2} M D_{R}^{\prime}+\frac{1}{4} M H_{R}^{\prime}$ \\
\hline$W_{P O M}$ & Maternal parent-offspring covariance & $\frac{1}{4} D_{R}+\frac{1}{4} M D_{R}^{\prime}$ \\
\hline$W_{P O P}$ & Paternal parent-offspring covariance & $\frac{1}{4} D_{R}$ \\
\hline
\end{tabular}

Maternal $M Z$ half sib-ship

$D_{R} \& H_{R}:$ See table 1 .

$$
M D_{R}^{\prime}=\sum_{i} 4 p_{i} q_{i}\left(m d_{i}+\left(q_{i}-p_{i}\right) m h_{i}\right)^{2} \quad M H_{R}^{\prime}=\sum_{i} 16 p_{i}^{2} q_{i}^{2} m h_{i}^{2}
$$


TABLE 3

Range of parameter values studied

Maternal effects-one character model

$$
\begin{array}{cccc}
\frac{m d}{d} & \frac{h}{d} & \frac{m h}{m d} & u \\
-1 \text { to } 2 & 0 \text { to } 2 & -1 \text { to } 2 & 0.1 \text { to } 0.9
\end{array}
$$

Maternal effects-two character model

$\begin{array}{ccccc}\frac{m d}{d} & \frac{h}{d} & \frac{m h}{m d} & u & p \\ -1 \text { to } 2 & 0 \text { to } 2 & -1 \text { to } 2 & 0.1 \text { to } 0.9 & 0.1 \text { to } 0.9\end{array}$

Sex linkage

$\begin{array}{ccc}\frac{d x^{\prime}}{d x} & \frac{h x}{d x} & u \\ 0 \cdot 2 \text { to } 5 & 0 \text { to } 2 & 0 \cdot 1-0 \cdot 9\end{array}$

frequencies. The range of parameter values studied is shown in table 3 . Taking first the one character model; over the normal range of dominance relationships from no dominance $(h=0)$ to complete dominance $\left(h_{i}=d_{i}\right)$ with each gene acting in the same direction whether contributing through a maternal effect or through the progeny's genotype ( $m d_{i}$ the same sign as $d_{i}$ and $m h_{i}$ the same sign as $\left.h_{i}\right)$, the maternal covariances $\left(\sigma_{a m}^{2}\right.$ and $\left.W_{P O M}\right)$ are always larger than their paternal counterparts $\left(\sigma_{a p}^{2}\right.$ and $\left.W_{P O P}\right)$. Furthermore, when the contribution through the maternal effect is similar $\left(d_{i}=d m_{i}\right.$, $\left.h_{i}=m h_{i}\right)$ to or greater $\left(d_{i}<m d_{i}, h_{i}<m h_{i}\right)$ than the direct effect through the progeny's genotype, the difference between the maternal and paternal half-sib covariances $\left(\sigma_{a m}^{2}-\sigma_{a p}^{2}\right)$ is greater than that between the maternal and paternal parent-offspring covariances $\left(W_{P O M}-W_{P O P}\right)$.

In the less likely situation where the contributions through the maternal effect $\left(m d_{i}\right.$ and $\left.m h_{i}\right)$ and through the progeny's genotype $\left(d_{i}\right.$ and $\left.h_{i}\right)$ are of opposite sign, the cross product terms of the one character model (table 1) become negative. In such circumstances, maternal covariances are often less than their paternal counterparts and in extreme cases the covariances may themselves be negative. These effects are more marked in the parentoffspring than in the half-sib covariances because of their greater relative contribution of cross-product terms. Similar effects may occur with genuine overdominance $\left(h_{i}>d_{i}\right.$ and $\left.m h_{i}>m d_{i}\right)$ or spurious overdominance arising from a linkage disequilibrium but these effects are only seen at extreme gene frequencies.

In summary, therefore, in the more likely situations, maternal effects that conform with the one character model will lead to greater maternal than paternal covariances and in most situations the half-sib covariances will be more sensitive to their presence than the parent-offspring covariances.

Because the two character model contains no cross-product terms $\left(d_{i} m d_{i}\right.$ and $\left.h_{i} m h_{i}\right)$ there are no circumstances in which the paternal covariances become greater than their maternal counterparts. In all circumstances, the difference between the maternal and paternal half-sib covariances is at least twice that between maternal and paternal parent-offspring covariances 
$\left(\left(\sigma_{a m}^{2}-\sigma_{a p}^{2}\right) \geqq 2\left(W_{P O M}-W_{P O P}\right)\right)$ and in the presence of dominance for the maternal effect ( $m h_{i}$ not zero) it is always greater than twice.

\section{Sex-Linked Loci}

Applying the model of Mather and Jinks $(1963,1971)$, we can write for a single sex-linked locus:

\begin{tabular}{lccccc} 
& \multicolumn{3}{c}{ Females } & \multicolumn{2}{c}{ Males } \\
Genotype & $A A$ & $A a$ & $a a$ & $A$ & $a$ \\
Frequency & $u^{2}$ & $2 u v$ & $v^{2}$ & $u$ & $v$ \\
Effect & $d x$ & $h x$ & $-d x$ & $d x^{\prime}$ & $-d x^{\prime}$
\end{tabular}

Summing over all such loci we can write the genetical contribution to the statistics derived from the MZ half-sib design in terms of their six components $D_{R}, H_{R}$ and

$$
\begin{aligned}
& D_{B}=\sum_{i} 4 u_{i} v_{i} d x_{i}^{\prime 2} \\
& D_{U}=\sum_{i} 4 u_{i} v_{i} d x_{i}^{\prime}\left[d x_{i}+\left(v_{i}-u_{i}\right) h x_{i}\right] \\
& D_{S}=\sum_{i} 4 u_{i} v_{i}\left[d x_{i}+\left(v_{i}-u_{i}\right) h x_{i}\right]^{2} \\
& H_{X}=\sum_{i} 16 u_{i}^{2} v_{i}^{2} h x_{i}^{2} .
\end{aligned}
$$

The contribution of heritable variation, including that of sex-linked loci, to the statistics obtained from an analysis of maternal and paternal MZ half-sibships is shown in table 4.

If we now consider male and female progeny separately, the contribution of sex-linked loci to the components of variance can also be written in terms of the four components $D_{B}, D_{U}, D_{S}$ and $H_{X}$. The contribution of heritable variation, including that of sex-linked loci, to the statistics derived from an analysis of variance of the female progeny of maternal and paternal MZ half-sibships is shown in table 5 and for the male progeny in table 6 .

Again it is instructive to substitute the more likely numerical relationships between $d x, d x^{\prime}$ and $h x$ and all possible gene frequencies into the statistics shown in tables 4,5 and 6 to see how they will behave under the conditions expected in practice. The range of parameter values studied is shown in table 3 . Considering initially the model in which the progeny sexes are analysed jointly, if there is no sex-limitation (i.e., $d x_{i}=d x_{i}^{\prime}$ ) and no dominance of sex-linked loci (i.e., $h x_{i}=0$ ) then $\sigma_{a m}^{2}>\sigma_{a p}^{2}$ and $W_{P O M}>$ $W_{P O P}$, a result which is indistinguishable from maternal effects. Furthermore, with $d x_{i}=d x_{i}^{\prime}$ and dominance of sex-linked genes (i.e., $h x_{i} \neq 0$ ) the relationships $\sigma_{a m}^{2}>\sigma_{a p}^{2}$ and $W_{P O M}>W_{P O P}$ still hold unless gene frequencies are extreme or the dominance ratio is very large. In these exceptional circumstances, $D_{U}$ may become negative in which case we may have $\sigma_{a m}^{2}<\sigma_{a p}^{2}$ although $W_{P O M} \geqq W_{P O P}$. 
TABLE 4

Sex-linkage-progeny sexes analysed jointly

Maternal MZ half-sibship

$\begin{array}{llc}\sigma_{a m}^{2} & \text { Maternal half-sib covariance } & \frac{1}{8} D_{R}+\frac{1}{8} D_{B}+\frac{1}{8} D_{U}+\frac{1}{32} D_{S} \\ \sigma_{b m}^{2} & \text { Full-sib covariance minus maternal } & \frac{1}{8} D_{R}+\frac{1}{16} H_{R}+\frac{1}{16} D_{S}+\frac{1}{32} H_{X} \\ & \text { half-sib covariance } & \\ \sigma_{w m}^{2} & \text { Within family variance } & \frac{1}{4} D_{R}+\frac{3}{16} H_{R}+\frac{3}{8} D_{B}-\frac{1}{8} D_{U}+\frac{5}{32} D_{S} \\ & & +\frac{3}{32} H_{X}\end{array}$

Paternal MZ half-sibship

$\sigma_{a p}^{2} \quad$ Paternal half-sib covariance

$\sigma_{h p}^{2} \quad$ Full-sib covariance minus paternal

$\frac{1}{8} D_{R}+\frac{1}{16} D_{S}$ half-sib covariance

$\frac{1}{8} D_{R}+\frac{1}{16} H_{R}+\frac{1}{8} D_{B}+\frac{1}{8} D_{U}+\frac{1}{32} D_{S}$ $+\frac{1}{32} H_{X}$

$\sigma_{w p}^{2} \quad$ Within family variance

$\frac{1}{4} D_{R}+\frac{3}{16} H_{R}+\frac{3}{8} D_{B}-\frac{1}{8} D_{U}+\frac{5}{32} D_{S}$ $+\frac{3}{32} H_{X}$

Full-sibship

$\sigma_{a}^{2}+\sigma_{b}^{2} \quad$ Full-sib covariance

$\frac{1}{4} D_{R}+\frac{1}{16} H_{R}+\frac{1}{8} D_{B}+{ }_{8}^{1} D_{U}+\frac{3}{32} D_{S}$ $+\frac{1}{32} H_{X}$

$\sigma_{a}^{2}+\sigma_{b}^{2}+\sigma_{w}^{2} \quad$ Total variance

$W_{P O M} \quad$ Maternal parent-offspring covariance

$W_{P O P}$

Paternal parent-offspring covariance

$\frac{1}{2} D_{R}+\frac{1}{4} H_{R}+\frac{1}{2} D_{B}+\frac{1}{4} D_{S}+\frac{1}{8} H_{X}$

$\frac{1}{4} D_{R}+\frac{1}{4} D_{U}+\frac{1}{8} D_{S}$

$\frac{1}{4} D_{R}+\frac{1}{4} D_{U}$

$$
\begin{array}{ll}
D_{B}=\sum_{i} 4 u_{i} v_{i} d x_{i}^{\prime 2} & D_{U}=\sum_{i} 4 u_{i} v_{i} d x_{i}^{\prime}\left[d x_{i}+\left(v_{i}-u_{i}\right) h x_{i}\right] \\
D_{S}=\sum_{i} 4 u_{i} v_{i}\left[d x_{i}+\left(v_{i}-u_{i}\right) h x_{i}\right]^{2} & H_{X}=\sum_{i} 16 u_{i}^{2} v_{i}^{2} h x_{i}^{2}
\end{array}
$$

N.B. These expectations assume that the variation due to the mean difference between sexes is removed before analysis.

\section{TABLE 5}

Sex-linkage-female progeny

\begin{tabular}{|c|c|c|}
\hline$\sigma_{a p Q}^{2}$ & Paternal half-sister covariance & $\frac{1}{8} D_{R}+\frac{1}{4} D_{S}$ \\
\hline$\sigma_{b p q}^{2}$ & $\begin{array}{l}\text { Sister covariance minus paternal } \\
\text { half-sister covariance }\end{array}$ & $\frac{1}{8} D_{R}+\frac{1}{16} H_{R}+\frac{1}{8} D_{S}+\frac{1}{8} H_{X}$ \\
\hline$\sigma_{w p q}^{2}$ & Within family variance of females & $\frac{1}{4} D_{R}+\frac{3}{16} H_{R}+\frac{1}{8} D_{S}+\frac{1}{8} H_{X}$ \\
\hline \multicolumn{3}{|l|}{ Full-sibship } \\
\hline$\sigma_{a \ell}^{2}+\sigma_{b \ell}^{2}$ & Sister covariance & $\frac{1}{4} D_{R}+\frac{1}{16} H_{R}+\frac{3}{8} D_{S}+\frac{1}{8} H_{X}$ \\
\hline$\sigma_{a q}^{2}+\sigma_{b q}^{2}+\sigma_{w 9}^{2}$ & Total female variance & $\frac{1}{2} D_{R}+\frac{1}{4} H_{R}+\frac{1}{2} D_{S}+\frac{1}{4} H_{X}$ \\
\hline$W_{P O M} 9$ & Maternal parent-daughter covariance & $\frac{1}{4} D_{R}+\frac{1}{4} D_{S}$ \\
\hline$W_{P O P Q}$ & Paternal parent-daughter covariance & $\frac{1}{4} D_{R}+\frac{1}{2} D_{U}$ \\
\hline
\end{tabular}

Maternal MZ half-sibship

$\begin{array}{lll}\sigma_{a m \&}^{2} & \text { Maternal half-sister covariance } & \frac{1}{8} D_{R}+\frac{1}{8} D_{S} \\ \sigma_{b m 8}^{2} & \text { Sister covariance minus maternal } & \frac{1}{8} D_{R}+\frac{1}{16} H_{R}+\frac{1}{4} D_{S}+\frac{1}{8} H_{X} \\ & \text { half-sister covariance } & \\ \sigma_{w m 8}^{2} & \text { Within family variance of females } & \frac{1}{4} D_{R}+\frac{3}{16} H_{R}+\frac{1}{8} D_{S}+\frac{1}{8} H_{X}\end{array}$

Paternal MZ half-sibship 
TABLE 6

Sex-linkage-male progeny

Maternal MZ half-sibship

\begin{tabular}{|c|c|c|}
\hline$\sigma_{a m \delta^{*}}^{2}$ & Maternal half-brother covariance & $\frac{1}{8} D_{R}+\frac{1}{2} D_{B}$ \\
\hline$\sigma_{b m \delta}^{2}$ & $\begin{array}{l}\text { Brother covariance minus maternal } \\
\text { half-brother coveriance }\end{array}$ & $\frac{1}{8} D_{R}+\frac{1}{16} H_{R}$ \\
\hline$\sigma_{w m \delta}^{2}$ & Within family variance of males & $\frac{1}{4} D_{R}+\frac{3}{16} H_{R}+\frac{1}{2} D_{B}$ \\
\hline \multicolumn{3}{|c|}{ Paternal MZ half-sibship } \\
\hline$\sigma_{a p \delta}^{2}$ & Paternal half-brother covariance & $\frac{1}{8} D_{R}$ \\
\hline$\sigma_{b p \delta}^{2}$ & $\begin{array}{l}\text { Brother covariance minus paternal } \\
\text { half-brother covariance }\end{array}$ & $\frac{1}{8} D_{R}+\frac{1}{16} H_{R}+\frac{1}{2} D_{B}$ \\
\hline$\sigma_{\text {wpo }}^{2}$ & Within family variance of males & $\frac{1}{4} D_{R}+\frac{3}{16} H_{R}+\frac{1}{2} D_{B}$ \\
\hline \multicolumn{3}{|l|}{ Full-sibship } \\
\hline$\sigma_{a \delta}^{2}+\sigma_{b \delta}^{2}$ & Brother covariance & $\frac{1}{4} D_{R}+\frac{1}{16} H_{R}+\frac{1}{2} D_{B}$ \\
\hline$\sigma_{a \delta}^{2}+\sigma_{b \delta}^{2}+\sigma_{w \delta}^{2}$ & Total male variance & $\frac{1}{2} D_{R}+\frac{1}{4} H_{R}+D_{B}$ \\
\hline$W_{\text {POM Oे }}$ & Maternal parent-son covariance & $\frac{1}{4} D_{R}+\frac{1}{2} D_{U}$ \\
\hline$W_{P O P \delta}$ & Paternal parent-son covariance & $\frac{1}{4} D_{R}$ \\
\hline
\end{tabular}

If we now consider the analyses of male and female progeny separately, under all but extreme levels of sex-limitation or genuine and spurious overdominance $\sigma_{a m \delta}^{2}<\sigma_{a p \delta}^{2}$ and $\sigma_{a m p}^{2}>\sigma_{a p \rho}^{2}$. And even in these extreme circumstances we never find both $\sigma_{a m q}^{2}>\sigma_{a p \uparrow}^{2}$ and $\sigma_{a m \delta}^{2}>\sigma_{a p \delta}^{2}$ which is the most likely result of maternal inheritance. Essentially the same patterns are shown by the parent-offspring covariances; in the most likely circumstances $W_{P O M}<W_{P O P}$ and $W_{P O M \delta}>W_{P O P \delta}$ and in no circumstances do we observe both $W_{P O M}>W_{P O P \text { q }}$ and $W_{P O M \delta}>W_{P O P \delta}$. Finally there are no circumstances in which sex-linkage will lead to the combined relationships

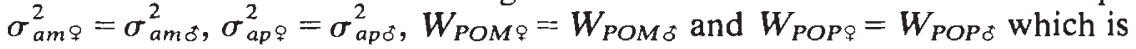
the expected norm for maternal inheritance. These inequalities which are characteristic of the presence of sex-linkage also extend to the three full-sib covariances (Mather and Jinks 1963, 1971). At a cruder level these inequalities also extend to the total variances of male and female offspring (tables 5 and 6) and also to their means. Nevertheless, it is ill advised to use these alone to detect sex-linkage as there are circumstances in which they will not differ when sex-linkage is present although other tests will still detect its presence. For example, if at the sex-linked loci there is no net directional dominance, that is, the dominance deviations are ambidirectional or there is a combination of no dominance $\left(h_{x}=0\right)$ and a sex-limitation in which $d x \simeq \sqrt{2} d x^{\prime}$, the total variances and means of the two sexes will not differ. But in these circumstances $\sigma_{a m}^{2}>\sigma_{a p}^{2}$ and $W_{P O M}>W_{P O P}$.

\section{Discussion}

It is clear from our algebraic models and numerical substitutions that over a wide range of conditions the MZ half-sib design can, in principle, detect heritable variation arising from maternal inheritance and sex-linked 
loci. Furthermore, providing the full analysis is used, in which corresponding statistics from male and female progeny analysed separately are compared, it is also possible to distinguish between maternal inheritance and sex-linkage. In practice, however, the sample sizes required to do so, except in those rare cases where either maternal effects or sex-linkages are the major source of variation (Nance, 1976), are likely, on past experience (Eaves and Jinks, 1972; Martin et al., 1978), to be well in excess of $100 \mathrm{MZ}$ half-sib families. More precise estimates covering all of the more likely situations that could be encountered in practice must await the outcome of computer simulations of the various models described in sections 2-4.

There have been several published reports in which the $M Z$ half-sib method has been used to provide evidence for the presence of maternal effects. The characters studied have included finger ridges (Reed et al., 1979), birth weight (Nance, 1976) and verbal IQ (Rose et al., 1980). It is not our intention to argue with the conclusion that these characters are subject to maternal effects-birthweight self-evidently is-but it must be pointed out that in none of these publications was it stated that heritable variation arising from sex-linked loci had been specifically ruled out. The tests used in these studies did not separate male and female progenies, so that while they would have detected both maternal effects and sex-linkage, they would not have been able to distinguish between them. And although the means and total variances of the two sexes did not differ in the study of Rose et al. (1980), if maternal half-sib and parent-offspring covariances are greater than their paternal counterparts it is not possible to rule out sex-linkage as a cause without carrying out tests that require that each of these statistics is derived separately for male and female progenies (section 4).

Given that the presence of maternal effects and the absence of sexlinkage has been confirmed by these tests, potentially the most informative use of the data is made by weighted least squares model fitting procedures (Mather and Jinks, 1971; Nance and Corey, 1976). Reference to tables 1 and 2 and our outline of the environmental parameters (section 2) shows that with the ten available statistics (i.e., the six statistics provided by an analysis of the progeny data, plus two from an analysis of the parental data and two parent-offspring covariances), $M D_{R}, M H_{R}$ and $H M H_{R}$ are confounded as are also $D_{R}, H_{R}, E_{B}^{\prime}, E_{B}^{\prime \prime}, D M D_{R}$ and any pair of $M D_{R}, M H_{R}$ and $H M H_{R}$. In this situation it is possible to obtain estimates of all of the parameters present in our two character model of maternal effects (i.e., $D_{R}$, $H_{R}, M D_{R}, M H_{R}, E_{W}, E_{B}^{\prime}$ and $E_{B}^{\prime \prime}$ ). However, because when the extra parameters required for the one character model are included a number of parameters are non-independent, it is necessary in practice to examine a variety of alternative models and subjectively select the most feasible (e.g., $D M D_{R}$ and $H M H_{R}$ are the only parameters that can have significantly negative estimates). Some other models for the environmental effects (for example that employed by Nance and Corey (1976)) lead to situations in which $D_{R}, H_{R}, E_{B}^{\prime}, E_{B}^{\prime \prime}, D M D_{R}$ and any pair of $M D_{R}, M H_{R}$ and $H M H_{R}$ are no longer confounded although of course $M D_{R}, M H_{R}$ and $H M H_{R}$ remain confounded. With data from further relationships, for example cousins, it may be possible to obtain independent estimates of all the parameters in both the one and two character models.

Although any reduction in the number or complexity of the components required to specify adequately the environmental sources of variation could 
improve the situation, in general the prospects of analysing maternal effects by fitting models to the ten statistics of the MZ half-sib design are not good. Furthermore, we do not believe that they can be improved by relying on unequal family sizes in the manner suggested by Nance and Corey (1976). Even if, because of the complexity of the environmental sources of variation, it is not possible to fit a satisfactory model it is still possible to test for maternal effects by direct comparisons of maternal and paternal half-sib correlations and of maternal and paternal parent-offspring correlations. If the two character model is appropriate, a comparison of the half-sib correlations is in general the more sensitive. But this is not true for the single character model unless the variation produced by the effect is as large as or larger than that produced by the direct effect of the progeny's own genotype, the maternal and direct effect act in the same direction and there is no genuine or spurious overdominance. As the maternal effect becomes a progressively smaller source of variation than the direct effect, so the comparison between the parent-offspring correlations becomes the more sensitive test. This is not only because the difference between the maternal and paternal parent-offspring correlations become larger than that between the maternal and paternal half-sib correlations but also because the parentoffspring correlations, being the larger provide a more sensitive test of the difference between them.

At the outset we noted that maternal effects could have many causes and that our genetic models were appropriate only where these had a heritable component. We shall, therefore, finally consider some consequence of a model in which the maternal effect depends solely upon the phenotype of the mother irrespective of its cause. A one character phenotypic model has been considered by Falconer (1964). In this model all environmental variation which affects the development of the parental phenotypes contributes to both maternal parent-offspring and half-sib covariances. In general this will lead to the maternal being greater than the paternal covariances but to an extent that cannot be quantified as in the case of the genotypic models.

In the two character phenotypic model the possibilities are more complex. For example, both the maternal parent-offspring and half-sib covariances would be reduced by any environmental variation which produces differences between the maternal twins for the character influencing the progeny phenotype that did not affect this character and the progeny phenotype in the same direction. On the other hand, any environmental variation which contributed to the covariance between maternal twins would contribute to the maternal half-sib covariance but would reduce the parent-offspring covariance. Thus on this model the value of the comparison of maternal and paternal half-sib correlations as a test of maternal effects is not reduced but that of maternal and paternal parentoffspring correlations is reduced. Once again, therefore, the $\mathrm{MZ}$ half-sib design emerges as the more robust test for maternal effects.

Turning now to sex-linked effects, if the data from the sexes are analysed separately, twenty statistics are available from the $\mathrm{MZ}$ half-sib design. It is possible to fit a model by the weighted least squares procedure to these statistics in which the four parameters defining sex-linked variation can be estimated simultaneously with all parameters previously discussed. That is $D_{S}, D_{B}, D_{U}$ and $H_{X}$ are not confounded with each other or with any combination of $D_{R}, H_{R}, M D_{R}, M H_{R}, D M D_{R}, H M H_{R}, E_{W}, E_{B}^{\prime}$ and $E_{B}^{\prime \prime}$. Thus 
if an inspection of data in which maternal effects are suspected reveals evidence of any difference between male and female estimates of parentoffspring or half-sib covariances, the adequacy of models can be examined in which both maternal effect parameters and sex-linkage parameters are included.

In the absence of weighted least squares procedures sex-linkage may be detected by making use of the oft quoted relationship $W_{P O M}=W_{P O P \&}>$ $W_{P O M}>W_{P O P \text { s }}$ although this relationship is only generally correct in the absence of dominance and sex-limitation of the sex-linked loci and with no maternal effects. Under these same conditions a comparison of half-sib covariances may detect sex-linkage for $\sigma_{a m \AA}^{2}>\sigma_{a p \&}^{2}>\sigma_{a m \S}^{2}>\sigma_{a p \delta^{+}}^{2}$. In practice the ordering of the correlations derived from these covariances may be complicated by dominance and sex-limitation of the sex-linked loci, by maternal effects and by inequalities in the total variance of males and females due to sex-linkage. In the presence of these complications model fitting would be the more enlightening approach to the detection and analysis of sex-linkage.

Acknowledgements.-This work is part of a research programme in Psychogenetics supported by the British Medical Research Council.

\section{REFERENCES}

BOCK, R. D., AND KOLAKOWSKI, D. 1973. Further evidence of sex-linked major gene influence on human spatial visualising ability. Amer. J. Human Genet., 25, 1-14.

EAVES, L. J., AND JINKS, J. L. 1972. Insignificance of evidence for differences in heritability of IQ between races and social classes. Nature, 240, 84-88.

FALCONER, D. S. 1964. Maternal effects and selection response. Genetics Today. Proceedings of the XI International Congress of Genetics. The Hague, Netherlands, September, 1963. Pergamon Press, Oxford.

KEMPTHORNE, O. 1957. An Introduction to Genetic Statistics. Wiley, New York.

MARTIN, N. G., EAVES, L. J., KEARSEY, M. J., AND DAVIES, P. 1978. The power of the classical twin study. Heredity, 40, 97-116.

MATHER, K., AND JINKS, J. L. 1963. Correlations between relatives arising from sex-linked genes. Nature, 198, 314-315.

MATHER, K., AND JINKS, J. L. 1971. Biometrical Genetics, 2nd Ed. Chapman and Hall, London.

NANCE, W. E. 1976. Genetic studies of the offspring of identical twins. A model for the analysis of quantitative inheritance in man. Acta. Genet. Med. Gemellol; 25, 103-113.

NANCE, W. E., AND COREY, L. A. 1976. Genetic models for the analysis of data from the families of identical twins. Genetics, 83,811-825

RAO, D. C., MORTON, N. E., AND YEE, S. 1974. Analysis of family resemblance. II. A linear model for familial correlation. Amer. J. Human. Genet., 26, 331-359.

REED, T., EVANS, M. M., NORTON, J. A., AND CHRISTIAN, J. C. 1979. Maternal effects on fingertip dermatoglyphics. Amer. J. Human. Genet., 31, 315-323.

ROBSON, E. B. 1955. Birth weight in cousins. Ann. Human. Genet., 19, 262-268.

ROSE, R. J., BOUGHMAN, J. A., COREY, L. A., NANCE, W. E., CHRISTIAN, J. C., AND KANG, K. w. 1980. Data from kinships of monozygotic twins indicate maternal effects on verbal intelligence. Nature, 283, 375-377. 